\title{
Phenolic constituents of Lamium album: Focus on isoscutellarein derivatives
}

\author{
Olívia R. Pereira ${ }^{\mathrm{a}, \mathrm{b}}$, Maria R.M. Domingues ${ }^{\mathrm{c}}$, Artur M.S. Silva ${ }^{\mathrm{c}}$, Susana M. Cardoso ${ }^{\mathrm{a}, \mathrm{d}, *}$ \\ ${ }^{a}$ CERNAS - Escola Superior Agrária, Instituto Politécnico de Coimbra, Bencanta, 3040-316 Coimbra, Portugal \\ b Departamento de Tecnologias de Diagnóstico e Terapêutica, Escola Superior de Saúde, Instituto Politécnico de Bragança, Av. D. Afonso V, 5300-121 Bragança, Portugal \\ c QOPNA - Departamento de Química, Universidade de Aveiro, 3810-193 Aveiro, Portugal \\ d CIMO - Escola Superior Agrária, Instituto Politécnico de Bragança, Campus de Santa Apolónia - Apartado 1038-5301-854, Bragança, Portugal
}

\section{A R T I C L E I N F O}

Article history:

Received 9 January 2012

Accepted 30 April 2012

Available online 11 May 2012

\section{Keywords:}

Lamium album

Verbascoside

Isoverbascoside

Isoscutellarein derivatives

High performance liquid chromatography

Electrospray ionization-mass spectrometry

\begin{abstract}
A B S T R A C T
Lamium album L. is an edible plant which is consumed raw or cooked, in particular in the Mediterranean and surrounding areas. It is also consumed as tea infusions and as a main component of food supplements, because of its pharmacological effects. Despite being consumed by humans for centuries, the chemical composition of $L$. album $\mathrm{L}$. is far from being understood. In this study, a purified ethanolic extract (PEEL) was prepared and further analyzed by high performance liquid chromatography and electrospray mass spectrometry. Overall, verbascoside accounted for approximately half of the phenolic content of the extract, but this also contained other bioactive phenolic compounds herein detected for the first time in the genus, namely isoscutellarein derivatives. The latter included isoscutellarein-7-O-allosyl $(1 \rightarrow 2)$ glucoside, its $O$-methyl derivative, three acetyl derivatives of isoscutellarein- 0 allosyl glucoside and one acetylated form of 0 -methylisoscutellarein-7-O-allosyl $(1 \rightarrow 2)$ glucoside. From those, the main isoscutellarein derivative was assigned to isoscutellarein-7-O-(6-O-acetyl- $\beta$-allosyl $)(1 \rightarrow 2)-\beta$-glucoside, as confirmed by NMR. Altogether, isoscutellarein derivatives accounted for almost $30 \%$ of PEEL phenolics. Since verbascoside and isoscutellarein derivatives are main components of $L$. album L. ethanolic extract, their possible association to the health benefits of the plant is discussed.
\end{abstract}

(c) 2012 Elsevier Ltd. All rights reserved.

\section{Introduction}

The genus Lamium L. (Family: Lamiaceae alt. Labiatae) comprises about 40 annual or perennial herb species native to the Old World, distributed in Europe, Asia and Africa.

Lamium album L. is a perennial herb commonly known as white dead nettle that has been used as emergency or famine food, particularly during the specific decades of starvation as an alternative nourishment in different countries such as Europe, China and Japan (Baranov, 1967; Luczaj, 2008; Sturtevants, 1919; Turner et al., 2011). In modern times, L. album L. is mainly consumed in the Mediterranean and surrounding areas for confection of local dishes (Heinrich, Müller, \& Galli, 2006). In fact, the young shoots, leaves and flowers of this plant are edible and consumed raw or cooked as a vegetable. The plant is also commonly used as an ingredient in several dishes including omelets, stews and roasts (Clifford, 2001). Moreover, white dead nettle is the base ingredient for important vegetarian dishes such as the "White Dead Nettle Frittata", "White Dead Nettle, Feta and Watermelon Salad" and the “Deadnettle soup" (Celnet, 2005; Harford, 2007).

\footnotetext{
* Corresponding author at: CERNAS - Escola Superior Agrária, Instituto Politécnico de Coimbra, Bencanta, 3040-316 Coimbra, Portugal. Tel.: +351 239 802940; fax: + 351273239802979 .

E-mail address: scardoso@esac.pt (S.M. Cardoso).
}

L. album L. is also used in teas and in food supplement preparations, the consumption of which is primarily associated to the plant health benefits. In particular, the consumption of food supplements enriched in L. album L. extracts are claimed to detoxify the organism, to prevent menstrual disorders, abdominal inflammation and musculoskeletal diseases (Xu, 2008) and to improve fat metabolism (Ninomiya et al., 2006).

Besides the above applications, the flowers of L. album L. are attractive to bees and other pollinating insects and hence, are frequently used in honey production (Denisow \& Bozek, 2008; Mihaly Cozmuta, Bretan, Mihaly Cozmuta, Nicula, \& Peter, in press).

During the last decades food health attributes have become an important issue of concern for consumers, clearly influencing their choices. In parallel, the search for food constituents related to health properties has incredibly raised. This provides the base knowledge to understand the beneficial properties of a particular food product and further stimulate consumers' interest in it. In the particular case of L. album L., the phenolic compounds have been closely associated with the antioxidant properties of the plant (Matkowski \& Piotrowska, 2006; Valyova, Dimitrova, Ganeva, Mihova Kapchina-Toteva, \& Petkova Yordanova, 2011), as well as to its remaining health benefits (Paduch et al., 2008; Paduch, Wójciak-Kosior, \& Matysik, 2007).

In this way, several L. album L. phenolic compounds have already been detected, which include the flavonoids quercetin, quercetin-3-Oglucoside, rutin, isoquercitrin, kaempferol-3-O-glucoside and tiliroside, 
the phenolic acids protocatechuic, chlorogenic, vanillic and caffeic and the phenylpropanoid glycoside ester derivatives lamalboside, acteoside and isoacteoside (Budzianowski \& Skrzypczak, 1995; Paduch et al., 2007; Yalcin \& Kaya, 2006). However despite that information, a detailed knowledge of the L. album L. phenolic constituents, as well as their content in the plant, is still missing. Hence, these two topics will be herein described in detail.

\section{Experimental}

\subsection{Chemicals}

The phenolic standards verbascoside, apigenin-7-0-glucoside, luteolin-7-0-glucoside and naringenin-7-O-glucoside were obtained from Extrasynthese (Genay Cedex, France). Gallic acid was obtained from Sigma Chemical Co (St Louis, MO, USA), while Folin-Ciocalteu reagent, $\mathrm{Na}_{2} \mathrm{CO}_{3}$, formic acid and ethanol were purchased from Panreac (Barcelona, Spain). n-Hexane, methanol and acetonitrile with HPLC purity were purchased from Lab-Scan (Lisbon, Portugal). Water was treated in a Milli-Q water purification system (TGI Pure Water Systems, USA). DMSO- $\mathrm{d}_{6}$ containing $0.03 \%$ of TMS was obtained from CortecNet (Paris, France).

\subsection{Plant material}

The $L$. album were purchased as a mixture of flowers, leaves and stems from O SEGREDO DA PLANTA - Produtos Naturais e Biológicos, Lda. (Seixal, Portugal). The plants have been cultivated under an organic regime and after collection, its aerial parts (flowers, leaves and stems) were dried in a ventilated incubator at 20 to $35^{\circ} \mathrm{C}$ for 3 to 5 days.

\subsection{Extraction of phenolic compounds}

The aerial parts (flowers, leaves and stems) of $L$ album ( $5 \mathrm{~g}$ ) were ground together and defatted three times with $150 \mathrm{~mL}$ of $n$-hexane. The residue was extracted with $150 \mathrm{~mL}$ of an $80 \%$ ethanol solution $(v / v)$ at room temperature, for $1 \mathrm{~h}$ and the resulting mixture was filtered. The residue was similarly re-extracted five times and the filtrated solutions were combined, concentrated, frozen at $-20{ }^{\circ} \mathrm{C}$ and freeze-dried. The dried extract (ethanolic extract) of $L$. album was stored under vacuum, in a desiccator in dark, for subsequent use (Pereira, Silva, Domingues, \& Cardoso, 2012). This procedure was performed in triplicate.

\subsection{Purification of phenolic compounds}

The ethanolic extracts were further purified for phenolic enrichment. For that, approximately $0.4 \mathrm{~g}$ of each ethanolic extract was dissolved in $15 \mathrm{~mL}$ of water and eluted in three Strata SPE C18-E cartridges (2 g, Waters, Milford, MA, USA). The cartridges were then washed three times with $30 \mathrm{~mL}$ of water, and the phenolic compounds were recovered by elution with $20 \mathrm{~mL}$ of methanol. The residue was concentrated, frozen at $-20^{\circ} \mathrm{C}$ and freeze-dried to give the purified ethanolic extract (PEEL) (Pereira et al., 2012).

\subsection{Quantification of total phenolic compounds}

Total concentration of phenolic compounds was determined according to the adapted Folin-Ciocalteu colorimetric method (Singleton \& Rossi, 1965). A mixture of $250 \mu \mathrm{L}$ of Folin-Ciocalteu reagent and $0.5 \mathrm{~mL}$ plant extract solution $(0.4 \mathrm{mg} / \mathrm{mL})$ was prepared. After $3 \mathrm{~min}, 1 \mathrm{~mL}$ of $\mathrm{Na}_{2} \mathrm{CO}_{3}(200 \mathrm{~g} / \mathrm{L})$ and $3.25 \mathrm{~mL}$ of milliQ water were added. The mixture was homogenized and incubated for $10 \mathrm{~min}$ at $70{ }^{\circ} \mathrm{C}$, and then kept at room temperature for $30 \mathrm{~min}$. The absorbance was measured at $700 \mathrm{~nm}$ and the amount of total phenolic compounds was expressed as gallic acid equivalent ( $\mathrm{mg} \mathrm{GAE}$ )/g dried weight of plant material using a calibration curve of gallic acid as standard ( 5 to
$37.5 \mu \mathrm{g} / \mathrm{mL}$ ). This procedure was performed at least in duplicate for the three PEEL samples.

\subsection{HPLC apparatus and chromatographic conditions}

The HPLC analysis was performed on a Varian 9010 separation module equipped with a PDA Varian Prostar detector and data acquisition and remote control of the HPLC system were done by Varian Star chromatography Workstation ${ }^{\circledR}$ (Lake Forest, CA, USA) software. The column used was a $250 \mathrm{~mm} \times 4 \mathrm{~mm}$ id, $5 \mu \mathrm{m}$ bead diameter, endcapped Nucleosil C18 (Macherey-Nagel) and its temperature was maintained at $30^{\circ} \mathrm{C}$.

Gradient elution was carried out with a mixture of $0.1 \%(v / v)$ of formic acid in water (solvent A) and acetonitrile (solvent B), which were degassed and filtered before use. The solvent gradient consisted of a series of linear gradients, starting from 10 to $20 \%$ of solvent B over $6 \mathrm{~min}, 20$ to $25 \%$ of solvent B over $6 \mathrm{~min}, 25$ to $40 \%$ over $30 \mathrm{~min}$, increasing to $45 \%$ at $50 \mathrm{~min}$ and to $100 \%$ of solvent B over 5 min decreasing to $10 \%$ of solvent B after 5 min followed by the return to the initial conditions. The flow rate used was $1 \mathrm{~mL} / \mathrm{min}$. For the HPLC analysis, the samples (10 mg) were dissolved in $2 \mathrm{~mL}$ of methanol, filtered through a $0.2 \mu \mathrm{m}$ Nylon membrane (Whatman) and $10 \mu \mathrm{L}$ of each solution was injected. The UV-vis spectra were recorded between 220 and $500 \mathrm{~nm}$ and the chromatographic profiles were recorded at $340 \mathrm{~nm}$.

\subsection{Identification and quantification of the phenolic compounds}

Identification of the compounds was performed by HPLC-DAD and ESI-MS analysis. The compounds were firstly identified according to the retention time and UV-vis spectra of the HPLC eluting peaks. After three manual collections, further characterization of the eluted compounds was accomplished by electrospray ionization mass spectrometry (ESI-MS and ESI-MS ${ }^{\mathrm{n}}$ ) using a Linear Ion trap LXQ mass spectrometer (ThermoFinnigan, San Jose, CA, USA), following the general procedure previously described (Pereira et al., 2012). Moreover, the most abundant isoscutellarein derivative (fraction 9) was further analyzed by NMR spectroscopy. To accomplish that, approximately $3 \mathrm{mg}$ of freeze-dried material of this HPLC fraction was dissolved in DMSO$\mathrm{d}_{6}$ and the ${ }^{1} \mathrm{H}$ and ${ }^{13} \mathrm{C}$ NMR spectra were recorded at $298 \mathrm{~K}$ on a Bruker Avance 500 spectrometer operating at $500.13 \mathrm{MHz}$ and $125.77 \mathrm{MHz}$, respectively. The phase sensitive ${ }^{1} \mathrm{H}$-detected $\left({ }^{1} \mathrm{H}^{13} \mathrm{C}\right)$ gHSQC (heteronuclear single quantum coherence, using gradient pulses for selection) spectrum was recorded with 216 transients over 256 increments (zero-filled to 512) and $2 \mathrm{~K}$ data points with spectral widths of $4500 \mathrm{~Hz}$ in $\mathrm{F}_{2}$ and $20 \mathrm{kHz}$ in $\mathrm{F}_{1}$. The repetition time was $1.9 \mathrm{~s}$. A cosine multiplication was applied in both dimensions. The delays were adjusted according to a coupling constant ${ }^{1} \mathrm{~J}(\mathrm{CH})$ of $147 \mathrm{~Hz}$. The gHMBC (heteronuclear multiple quantum coherence, using gradient pulses for selection) spectrum was recorded with 240 transients over 256 increments (zero-filled to $1 \mathrm{~K}$ ) and $2 \mathrm{~K}$ data points with spectral widths of $4500 \mathrm{~Hz}$ in $F_{2}$ and $25 \mathrm{kHz}$ in $F_{1}$. The repetition time was $1.9 \mathrm{~s}$. A sine multiplication was applied in both dimensions. The low-pass $J$-filter of the experiment was adjusted for an average coupling constant ${ }^{1} \mathrm{~J}(\mathrm{CH})$ of $147 \mathrm{~Hz}$ and the long-range delay utilized to excite the heteronuclear multiple quantum coherence was optimized for $7 \mathrm{~Hz}$.

Taking into account the nature of the phenolic compounds (phenylethanoids and flavones), their quantification was performed at $340 \mathrm{~nm}$ (Galvez, Martin-Cordero, Houghton, \& Ayuso, 2005) by the external standard method. The detection and quantification limits (LOD and $\mathrm{LOQ}$, respectively) were determined from the parameters of the calibration curves represented in Table 1, being defined as 3.3 and 10 times the value of the regression error divided by the slope, respectively (Ermer \& Miller, 2005; Snyder, Kirkland, \& Dolan, 2010).

Fractions 2 and 3 (verbascoside, isoverbascoside) were quantified using verbascoside as a reference compound. Apigenin-7-0-glucoside was used to quantify fractions 4 [isoscutellarein-7-0-allosyl $(1 \rightarrow 2$ ) 
Table 1

Linearity, LOD and LOQ of four standard compounds used as references.

\begin{tabular}{|c|c|c|c|c|c|c|c|}
\hline Standard compound & $\begin{array}{l}\text { Range concentration } \\
(\mu \mathrm{g} / \mathrm{mL})\end{array}$ & $\mathrm{n}^{\mathrm{a}}$ & $\begin{array}{l}\text { Slope }{ }^{\mathrm{b}} \\
\text { (area counts/mg) }\end{array}$ & $\begin{array}{l}\text { Intercept }{ }^{\mathrm{b}} \\
\text { (area counts/mg) }\end{array}$ & $\mathrm{R}^{2}$ & $\begin{array}{l}\text { LOD } \\
(\mu \mathrm{g} / \mathrm{mL})\end{array}$ & $\begin{array}{l}\text { LOQ } \\
(\mu \mathrm{g} / \mathrm{mL})\end{array}$ \\
\hline L-70-G & $45-473$ & 5 & $763( \pm 1) \times 10^{4}$ & $13( \pm 9) \times 10^{4}$ & 0.9967 & 32.5 & 98.4 \\
\hline Verb & $44-700$ & 5 & $166( \pm 6) \times 10^{4}$ & $6( \pm 2) \times 10^{3}$ & 0.9985 & 31.9 & 96.7 \\
\hline A-70-G & $40-500$ & 5 & $151( \pm 7) \times 10^{5}$ & $-6( \pm 1) \times 10^{5}$ & 0.9992 & 17.3 & 52.4 \\
\hline N-70-G & $5-68$ & 5 & $230( \pm 8) \times 10^{4}$ & $-2( \pm 6) \times 10^{3}$ & 0.9990 & 2.7 & 8.2 \\
\hline
\end{tabular}

L-70-G, luteolin-7-O-glucoside; Verb, verbascoside; A-7O-G, apigenin-7-O-glucoside; N-70-G, naringenin-7-0-glucoside.

a Number of points used for the regression of standard solutions. Injections were done in triplicate.

b The standard deviation in the slope and intercept of the regression line is shown in parentheses.

glucoside], 5 [isoscutellarein-7-0-(6-0-acetylallosyl) $(1 \rightarrow 6)$ glucoside], 7 [isoscutellarein-7-0-(6-O-acetylallosyl $)(1 \rightarrow 2)$ glucoside isomer], 9 [isoscutellarein-7-0-(6-O-acetylallosyl) $(1 \rightarrow 2)$ glucoside], 10 [4'-Omethylisoscutellarein-7-O-allosyl $(1 \rightarrow 2)$ glucoside], 11 [4'-O-methylisoscutellarein-7-O-(6-O-acetylallosyl) $(1 \rightarrow 2)$ glucoside], 8 (apigenin7-O-glucoside) and 12 (apigenin-7-O-rutinoside). Fraction 6 (luteolin7-O-glucoside) was quantified with luteolin-7-O-glucoside while naringenin-7-O-glucoside was used as the reference for quantification of phenolic compounds in fraction 13 (naringenin-7-0-rutinoside).

\section{Results and discussion}

The purified ethanolic extract of L. album (PEEL) represented $13 \%$ of the dried plant mass and its total phenolic compounds accounted for $192.5 \pm 10.3 \mathrm{mg} \mathrm{GAE} / \mathrm{g}$ of PEEL, which corresponds to a recovery of $24.24 \mathrm{mg} \mathrm{GAE} / \mathrm{g}$ of dried plant. This result is lower than that reported by Matkowski and Piotrowska (2006) (32.8 $\pm 4.0 \mathrm{mg} \mathrm{GAE} / \mathrm{g}$ of dried plant) and differences can be ascribed to various factors, such as different agronomic or extraction conditions.

\subsection{Identification of phenolic compounds in PEEL}

As can be observed in Fig. 1 and Table 2, the present study allowed identification of thirteen phenolic components in PEEL, which comprised flavones, phenylethanoid isomers and one flavanone. From the above compounds, derivatives of the uncommon flavone isoscutellarein were detected for the first time in the Lamium genus, and thus, their identification will be described below in detail.

\subsubsection{Isoscutellarein derivatives}

Overall, six isoscutellarein derivatives could be detected in PEEL (Table 2 and Fig. 2). These compounds, eluted in fractions 4, 5, 7, 9, 10 and 11 , showed characteristic UV spectra with maxima at 278 , 302 and $333 \mathrm{~nm}$, which is in agreement with that described for isoscutellarein glucosides (Innocenti et al., 2007; Sahin, Ezer, \& Calis, 2006; Saracoglu, Harput, \& Ogihara, 2004). Notably, this is the first study reporting this flavonoid aglycone class in the Lamium genus, refuting previous chemotaxonomic studies of the plant (TomásBarberán, Grayer-Barkmeijer, Gil, \& Harborne, 1988).

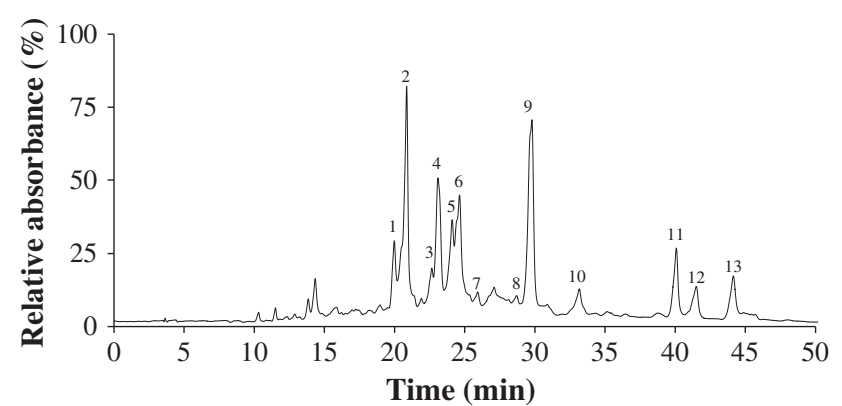

Fig. 1. Chromatographic profile at $340 \mathrm{~nm}$ of purified ethanolic extract of Lamium album $\mathrm{L}$.
In more detail, the phenolic compound of fraction 4 corresponded to isoscutellarein-7-0-allosyl $(1 \rightarrow 2)$ glucoside. This compound was detected in the ESI-MS spectrum as a $[\mathrm{M}-\mathrm{H}]^{-}$ion at $m / z 609$, and its main product ion $(\mathrm{m} / \mathrm{z} 285)$ was formed by the loss of $324 \mathrm{Da}$, which indicates an $\mathrm{O}$-glycosylation on a phenolic hydroxyl with a dihexoside (Ferreres, Llorach, \& Gil-Izquierdo, 2004). Moreover, the product ion $[\mathrm{M}-\mathrm{H}-180]^{-}$at $\mathrm{m} / \mathrm{z} 429$ indicated the $1 \rightarrow 2$ glycosylation between the sugars (Ferreres et al., 2004; Petreska et al., 2011). Note that this compound has been previously described to occur in genus Stachys and Sideritis, both belonging to the same subfamily (Lamioideae) as Lamium (Ferreres et al., 2004; Petreska et al., 2011; Tomás-Barberán, Francisco, Gil, Ferreres, \& Tomás-Lorente, 1992).

In a similar way, the compound eluting in fraction $10\left([\mathrm{M}-\mathrm{H}]^{-}\right.$ion at $m / z 623$ ) was tentatively assigned as the $4^{\prime}-0$-methyl derivative of the previous compound. Besides the characteristic base peak in $\mathrm{MS}^{2}$ spectrum at $m / z 299(-324 \mathrm{Da})$ and the product ions [M-H-162 $]^{-}$ (ion at $m / z 461$ ) and $[\mathrm{M}-\mathrm{H}-180]^{-}$(ion at $m / z 443$ ), due to loss of the hexose as residue and as unit, respectively, this compound also showed the simultaneous loss of the disaccharide moiety and a methyl group (ion at $m / z 284$ ), which is in agreement with the pattern fragmentation of $4^{\prime}$-O-methylisoscutellarein-7-O-allosyl $(1 \rightarrow 2)$ glucoside, recently detected in Stachys and Sideritis genus (Karioti, Bolognesi, Vincieri, \& Bilia, 2010; Petreska et al., 2011)

Isoscutellarein acetyl derivatives were also found in PEEL (fractions $5,7,9$ and 11), as confirmed by the initial loss of $42 \mathrm{Da}$ in their MS ${ }^{2}$ spectra. From those, the isomeric compounds (MW $652 \mathrm{Da}$ ) which eluted in the first three fractions were the acetyl derivatives of isoscutellarein-O-allosyl $(1 \rightarrow 2$ )glucoside (compound of fraction 4 ) and of $4^{\prime}$-O-methylisoscutellarein-7-O-allosyl $(1 \rightarrow 2)$ glucoside (compound of fraction 10).

The $\mathrm{MS}^{2}$ spectrum of the major acetylated isomer of isoscutellarein0 -allosyl $(1 \rightarrow 2)$ glucoside, eluted in fraction $9\left([\mathrm{M}-\mathrm{H}]^{-}\right.$ion at $\left.m / z 651\right)$, showed a base peak at $m / z 285\left([\mathrm{M}-\mathrm{H}-324-42]^{-}\right)$, which is indicative for $\mathrm{O}$-acetylglycosylation onto the phenolic hydroxyl groups (Petreska et al., 2011). Moreover, the intermediate ion $[\mathrm{M}-\mathrm{H}-42-180]^{-}$at $\mathrm{m} / \mathrm{z}$ 429 was indicative of an acetyl group on the external sugar (Karioti et al., 2010). Overall, the fragmentation pattern of this compound corresponded to that of isoscutellarein-7-0-(6-0-acetylallosyl $)(1 \rightarrow 2)$ glucoside. Moreover, this assignment was confirmed by NMR spectroscopy, as all the ${ }^{1} \mathrm{H}$ NMR and ${ }^{13} \mathrm{C}$ NMR signals (Table 3 ) were consistent with that isoscutellarein derivative (Albach, Grayer, Jensen, Ozgokce, \& Veitch, 2003; Gabrieli, Kefalas, \& Kokkalou, 2005; Sahin et al., 2006).

Regarding the remaining isoscutellarein acetyl derivatives (fractions 5 and 7), they should have distinct 0 -acylation and/or glycosylation with respect to the previous compound. At this point, the exact features of those groups could not be determined. Even so, it is possible to predict that the isomer in fraction 7 also contains a $1 \rightarrow 2$ glycosylation, as dictated by the occurrence of the product ion at $m / z 429$ ([M-H$\left.180-42]^{-}\right)$in its $\mathrm{MS}^{2}$ spectrum. This isomer must correspond to isoscutellarein 7-O-(4-O-acetylallosyl $)(1 \rightarrow 2)$ glucoside or to isoscutellarein 7-O-(2-O-acetylallosyl) $(1 \rightarrow 2)$ glucoside, since acylations of flavonoid glycosides can also occur in 2- and 4-positions of the hexose (Cuyckens \& Claeys, 2004). On the other hand, the 0 -glycosylation type of the 
Table 2

Identification of HPLC eluting fractions by HPLC-DAD, ESI-MS and ESI-MS ${ }^{\mathrm{n}}$ from ethanolic extract of Lamium album L.

\begin{tabular}{|c|c|c|c|c|c|}
\hline Peak & $\begin{array}{l}\mathrm{RT} \\
(\min )\end{array}$ & $\lambda_{\max }$ & {$[\mathrm{M}-\mathrm{H}]^{-}$} & $\begin{array}{l}\text { Main fragment } \\
\text { ESI-MS }^{\mathrm{n}}\end{array}$ & Compound \\
\hline 1 & 20.0 & $\begin{array}{l}254,267 \\
345\end{array}$ & - & ( & Luteolin derivative \\
\hline 2 & 20.9 & 290,329 & 623 & $\begin{array}{l}\operatorname{MS}^{2} \text { [623]: } 477 \text { (2\%), 461; } \text { MS }^{3} \text { [461]: } 315 \text { (100\%), } 297 \text { (10\%), } \\
135 \text { (30\%) }\end{array}$ & Verbascoside \\
\hline 3 & 22.7 & 290,328 & 623 & $\begin{array}{l}\text { MS }^{2} \text { [623]: } 477 \text { (2\%), } 461 \text { (100\%), } 299 \text { (5\%); } \text { MS }^{3} \text { [461]: } 315 \text { (100\%), } \\
297 \text { (10\%), } 161 \text { (3\%), } 135 \text { (30\%); } \text { MS }^{4}[315]: 135\end{array}$ & Isoverbascoside \\
\hline 4 & 23.1 & $\begin{array}{l}275,302, \\
333\end{array}$ & 609 & $\begin{array}{l}\text { MS }^{2} \text { [609]: } 489 \text { (2\%), } 447 \text { (20\%), } 429(40 \%) 285(100 \%) \\
\text { MS }^{3} \text { [429]: } 285 \text { (100\%), } 284(10 \%) ; M^{4}[285]: 267(5 \%) \\
257 \text { (20\%), } 241(100 \%), 213(40 \%), 199(3 \%), 197(4 \%) \\
191(10 \%) ; \text { MS }^{5}[241]: 213(100 \%), 197(40 \%), 185(45 \%) \\
145 \text { (10\%) }\end{array}$ & Isoscutellarein-7-O-allosyl $(1 \rightarrow 2)$ glucoside \\
\hline 5 & 24.1 & $\begin{array}{l}275,302, \\
333\end{array}$ & 651 & $\begin{array}{l}\text { MS }^{2} \text { [651]: } 609 \text { (100\%), } 285(2 \%) ; \mathrm{MS}^{3}[609]: 489(4 \%) 447(85 \%), \\
285 \text { (100\%); } \mathrm{MS}^{4}[447]: 285 ; \mathrm{MS}^{5}[285]: 267(3 \%), 243(60 \%), \\
241(100 \%), 217(35 \%), 199(43 \%), 175(40 \%), 151(3 \%)\end{array}$ & Isoscutellarein-7-O-(6-O-acetylallosyl $)(1 \rightarrow 6)$ glucoside \\
\hline 6 & 24.6 & $\begin{array}{l}254,267 \\
345\end{array}$ & 447 & $\begin{array}{l}\text { MS }^{2} \text { [447]: } 285 ; \mathrm{MS}^{3} \text { [285]: } 243 \text { (5\%), } 241 \text { (100\%), } 217 \text { (60\%), } \\
199 \text { (60\%), } 175(60 \%)\end{array}$ & Luteolin-7-O-glucoside \\
\hline 7 & 25.9 & $\begin{array}{l}275,302, \\
333\end{array}$ & 651 & $\begin{array}{l}\operatorname{MS}^{2} \text { [651]: } 609 \text { (100\%), } 591 \text { (10\%), } 447 \text { (2\%), } 429 \text { (5\%), } 285 \text { (20\%); } \\
\text { MS }^{3} \text { [609]: } 447 \text { (5\%), } 429 \text { (30\%), } 285(100 \%)\end{array}$ & $\begin{array}{l}\text { Isoscutellarein-7-O-(6-O-acetylallosyl })(1 \rightarrow 2) \text { glucoside } \\
\text { isomer }\end{array}$ \\
\hline 8 & 28.7 & 266,342 & 431 & $\begin{array}{l}\operatorname{MS}^{2} \text { [431]: 269; } \mathrm{MS}^{3} \text { [269]: } 227 \text { (100\%), } 225 \text { (90\%), } 199 \text { (85\%), } \\
180 \text { (95\%) }\end{array}$ & Apigenin-7-O-glucoside \\
\hline 9 & 29.8 & $\begin{array}{l}275,302, \\
333\end{array}$ & 651 & $\begin{array}{l}\operatorname{MS}^{2}[651]: 609(15 \%), 591(10 \%), 447(7 \%), 429(45 \%), 285(100 \%) ; \\
\text { MS }^{3} \text { [429]: } 285 ; \text { MS }^{4}[285]: 257(30 \%), 241(100 \%), 213(30 \%), 191(7 \%), \\
171 \text { (4\%) }\end{array}$ & Isoscutellarein-7-O-(6-O-acetylallosyl) $(1 \rightarrow 2)$ glucoside \\
\hline 10 & 33.2 & $\begin{array}{l}275,305 \\
327\end{array}$ & 623 & $\begin{array}{l}\text { MS }^{2} \text { [623]: } 461 \text { (15\%), } 443 \text { (3\%), } 299 \text { (100\%), } 284 \text { (10\%); } \text { MS }^{3} \text { [461]: 299; } \\
\text { MS }^{4} \text { [299]: } 284 \text { (100\%), } 255 \text { (1\%), } 240(4 \%)\end{array}$ & 4'-O-Methylisoscutellarein-7-O-allosyl $(1 \rightarrow 2)$ glucoside \\
\hline 11 & 40.1 & $\begin{array}{l}275,305 \\
327\end{array}$ & 665 & $\begin{array}{l}\operatorname{MS}^{2} \text { [665]: } 623 \text { (15\%), } 461(10 \%), 443(5 \%), 299(100 \%), 284(15 \%) ; \\
\text { MS }^{3} \text { [461]: } 299 ; \mathrm{MS}^{4} \text { [299]: } 284(100 \%), 255(1 \%), 256(1 \%), 240(5 \%) \\
227 \text { (1\%); } \mathrm{MS}^{5}[284]: 283(100 \%), 256(25 \%), 227(20 \%), 228(19 \%) \\
212(8 \%), 200(4 \%), 150(1 \%), 137(7 \%)\end{array}$ & $\begin{array}{l}\text { 4'-O-Methylisoscutellarein-7-O-(6-O-acetylallosyl })(1 \rightarrow 2) \\
\text { glucoside }\end{array}$ \\
\hline 12 & 41.5 & 266,342 & 577 & $\begin{array}{l}\text { MS }^{2} \text { [577]: } 431 \text { (1\%), } 307 \text { (3\%), } 269 \text { (100\%); } \text { MS }^{3} \text { [269]: } 227 \text { (10\%), } \\
225 \text { (100\%), } 201 \text { (15\%), } 183 \text { (2\%), } 151(10 \%), 149(15 \%)\end{array}$ & Apigenin-7-O-rutinoside \\
\hline 13 & 44.2 & - & 579 & $\begin{array}{l}\mathrm{MS}^{2} \text { [579]: } 307 \text { (75\%), } 271(100 \%) ; \mathrm{MS}^{3}[307]: 247 \text { (25\%), } 205(20 \%), \\
187 \text { (25\%), } 175 \text { (3\%), } 163 \text { (50\%), } 145 \text { (100\%); } \mathrm{MS}^{3}[271]: 177(10 \%), \\
151 \text { (100\%) }\end{array}$ & Naringenin-7-O-rutinoside \\
\hline
\end{tabular}

Peak 1 assignment was only based on UV spectra, which corresponded to that of luteolin.

isomer eluted in fraction 5 differs from that of the other two. Probably this is a $1 \rightarrow 6$ glycosidic type ligation, since the product ion $[\mathrm{M}-\mathrm{H}-42-162]^{-}$(at $\mathrm{m} / \mathrm{z} 447$ ) was prevalent while $[\mathrm{M}-\mathrm{H}-42-$ $180]^{-}$or $[\mathrm{M}-\mathrm{H}-180]^{-}$product ion was not observed in $\mathrm{MS}^{\mathrm{n}}$ experiments (Ferreres et al., 2004). To our knowledge, isoscutellarein-7-O$(6-0$-acetylallosyl $)(1 \rightarrow 2)$ glucoside isomers with distinct 0 -acylation and/or glycosylation positions have not been described in literature so far.

The acetylated form of 4'-O-methylisoscutellarein-7-O-allosyl $(1 \rightarrow 2)$ glucoside (MW $666 \mathrm{Da}$ ) was found in fraction 11. Accordingly, the MS spectrum of this fraction showed the $[\mathrm{M}-\mathrm{H}]^{-}$at $m / z 665$ and its $\mathrm{MS}^{2}$ spectrum showed high relative abundance ions at $\mathrm{m} / \mathrm{z} 299$ and at $\mathrm{m} / \mathrm{z}$ $623\left([\mathrm{M}-\mathrm{H}-42]^{-}\right)$(correspondent to methylisoscutellarein). Moreover, the fragmentation pattern of the latter ion was similar to that described for the 4'-O-methylisoscutellarein-7-O-allosyl $(1 \rightarrow 2)$ glucoside (fraction 10).

\subsubsection{Other phenolic compounds}

Besides the isoscutellarein derivatives previously described, PEEL also contained glycosides of common flavones, namely luteolin-7-Oglucoside (fraction 6), apigenin-7-0-glucoside (fraction 8), apigenin7-0-rutinoside (fraction 12), the flavanone naringenin-7-0-rutinoside (fraction 13) and two phenylethanoid glycosides (verbascoside and isoverbascoside, in fractions 2 and 3, respectively). The latter showed UV data and fragmentation pathway similar to that described in literature (Li, Liu, Liu, Tsao, \& Liu, 2009). In particular, the MS ${ }^{2}$ of their molecular ion $\left([\mathrm{M}-\mathrm{H}]^{-}\right.$at $\left.m / z 623\right)$ showed a base peak product ion resultant from the loss of caffeoyl $\left(-162 \mathrm{Da}\right.$, ion at $\mathrm{m} / z$ 461) while the MS ${ }^{3}$ data of this latter ion supported the main loss of a rhamnose unit (ion at $m / z 315$ ). Note that the phenylethanoid glycoside eluting in the most intense HPLC peak (fraction 2) corresponded to verbascoside, which has previously been described to occur in several Lamium species, including in L. album (Budzianowski \& Skrzypczak, 1995). Still, to

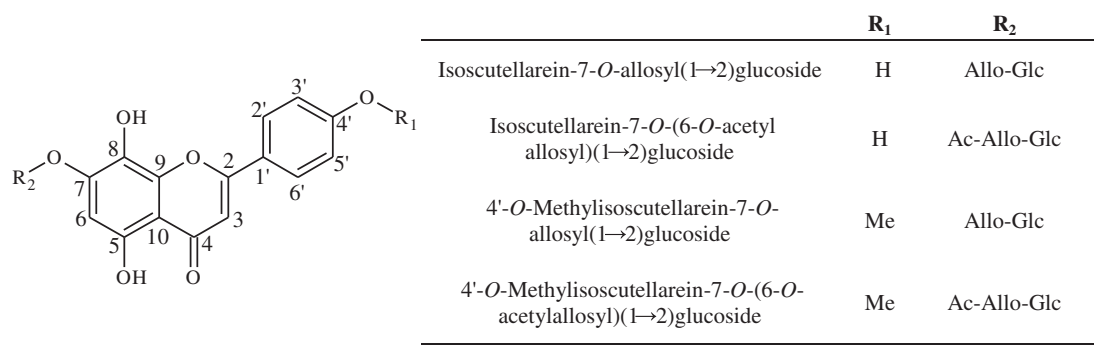

Allo, Allosyl unit; Glc, Glucosyl unit; Ac, Acetyl unit; Me, Methyl unit

Fig. 2. Main features of isoscutellarein derivatives found in purified extract of Lamium album L. 
Table 3

${ }^{13} \mathrm{C}$ and ${ }^{1} \mathrm{H}$ NMR spectral data for the compound isoscutellarein-7-O-(6-O-acetylallosyl) $\left(1 \rightarrow 2\right.$ )glucoside obtained from purified phenolic extract of Lamium album L. (in DMSO- $\mathrm{d}_{6}$ ).

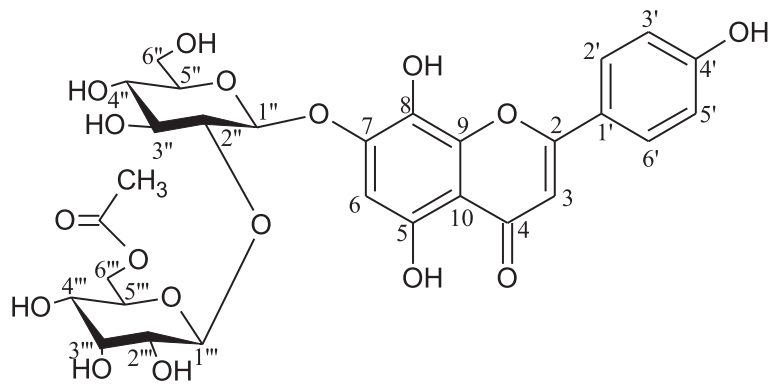

\begin{tabular}{|c|c|c|c|c|c|}
\hline Atom & ${ }^{13} \mathrm{C}$ & ${ }^{1} \mathrm{H}$ & Atom & ${ }^{13} \mathrm{C}$ & ${ }^{1} \mathrm{H}$ \\
\hline \multicolumn{3}{|c|}{ Aglycone } & \multicolumn{3}{|c|}{ Glucose $^{\mathrm{a}}$} \\
\hline 2 & 164.1 & - & $1 "$ & 100.0 & $5.09(\mathrm{~d}, J=7.4 \mathrm{~Hz})$ \\
\hline 3 & 102.6 & 6.85 & $2 "$ & 82.6 & $3.59(' \mathrm{t}$ ', $J=8.3 \mathrm{~Hz})$ \\
\hline 4 & 182.4 & - & 3" & 75.6 & - \\
\hline 5 & 152.2 & 12.38 & 4" & 69.2 & - \\
\hline 6 & 100.0 & 6.70 & $5 "$ & 77.1 & - \\
\hline 7 & 150.5 & - & 6" & 60.5 & $3.74(\mathrm{dd}, J=10.6$ and $5.3 \mathrm{~Hz})$ \\
\hline 8 & 127.5 & 7.95 & \multicolumn{3}{|r|}{ (1) } \\
\hline 9 & 143.7 & - & 1"' & 102.6 & $4.92(\mathrm{~d}, J=7.9 \mathrm{~Hz})$ \\
\hline 10 & 105.5 & - & 2"' & 71.4 & - \\
\hline $1^{\prime}$ & 121.2 & - & 3"' & 70.8 & $3.92-3.90$ \\
\hline $2^{\prime}$ & 128.7 & $8.00(\mathrm{~d}, J=8.5 \mathrm{~Hz})$ & 4"' & 66.8 & $3.92-3.90$ \\
\hline $3^{\prime}$ & 115.9 & $6.95(\mathrm{~d}, J=8.5 \mathrm{~Hz})$ & 5"' & 71.5 & $3.88-3.86$ \\
\hline $4^{\prime}$ & 161.3 & - & 6"' & 63.5 & $4.02(\mathrm{~d}, J=2.7 \mathrm{~Hz})$ \\
\hline $5^{\prime}$ & 115.9 & $6.95(\mathrm{~d}, J=8.5 \mathrm{~Hz})$ & $\mathrm{OAC}$ & & \\
\hline \multirow[t]{2}{*}{$6^{\prime}$} & 128.7 & $8.00(\mathrm{~d}, J=8.5 \mathrm{~Hz})$ & & 20.5 & 1.88 \\
\hline & & & & 170.3 & - \\
\hline
\end{tabular}

${ }^{a}$ The $\mathrm{OH}$ groups of the sugar moiety appear at: $5.25(\mathrm{~d}, J=5.5 \mathrm{~Hz}, 1 \mathrm{H}), 5.16$ $(\mathrm{d}, J=4.2 \mathrm{~Hz}, 1 \mathrm{H}),, 5.02(\mathrm{~d}, J=3.4 \mathrm{~Hz}, 1 \mathrm{H}), 4.83(\mathrm{~d}, J=7.8 \mathrm{~Hz}, 1 \mathrm{H}), 4.74(\mathrm{t}, J=5.5 \mathrm{~Hz}, 1 \mathrm{H})$.

our knowledge, isoverbascoside (fraction 3 ) is herein described for the first time in this species.

\subsection{Quantification of phenolic compounds in PEEL}

The quantification of the distinct phenolic compounds in PEEL extract was carried out using calibration curves of each available standard. Table 1 shows typical analytical parameters including the limits of detection and quantification (LOD and LOQ, respectively), the calibration curves, the linearity and the regression coefficient $\left(\mathrm{R}^{2}\right)$.

The quantified phenolic compounds in the ethanolic extract of L. album accounted for $500.7 \pm 50.0 \mathrm{mg} / \mathrm{g}$ of extract (Table 4 ), that is equivalent to $14.9 \mathrm{mg} / \mathrm{g}$ of dry plant. This extract was mainly rich in verbascoside, which, together with isoverbascoside, accounted for approximately $55 \%$ of the total phenolic content of PEEL. Also important, the glucosyl-isoscutellarein derivatives of this extract were present in appreciable amounts (total of $27 \%$ ), mostly in the acetylated form (18\%). Still note that accurate quantification of these compounds can be impaired, as optimum peak separation was not achieved for all the compounds and apigenin-7-O-glucoside was used as a reference for isoscutellarein derivative quantification, instead of the exact reference compounds.

The high content of the phenylethanoid glycosides verbascoside and isoverbascoside in the ethanolic extract of $L$. album suggests that medicinal activities claimed to this plant can be associated with these compounds. In fact, several studies reported important activities for verbascoside, including antioxidant and free radical scavenging capacity, neuroprotective, hepatoprotective, analgesic, cytotoxic, antimicrobial, anti-inflammatory and beneficial effects on the cardiovascular system. Most of these activities are also ascribed to isoverbascoside (Fu, Pang, \& Wong, 2008; Isacchi et al., 2011; Korkina, 2007; Kostyuk, Potapovich, Suhan, de Luca, \& Korkina, 2011; Morikawa et al., 2010). Moreover, it is important to highlight that despite the presence of lower amounts of isoscutellarein derivatives as compared to those of phenylethanoid glycosides, these can also be key components on the ethnopharmacological effects of the plant. Indeed, for the last decades, isoscutellarein derivatives have also been described to exert important beneficial activities as antiviral, antioxidant, cytotoxic, antinociceptive, anti-inflammatory and inhibitory activity against osteoclastogenesis (Kupeli, Sahin, Yesilada, Calis, \& Ezer, 2007; Nagai, Miyaichi, Tomimori, Suzuki, \& Yamada, 1992; Yang et al., 2003; Yoon, Jeong, Hwang, Ryu, \& Kim, 2007).

\section{Conclusions}

The phenolic composition of the purified ethanolic extract of aerial parts of L. album was assessed by a combined method using HPLC-DAD and ESI-MS. The extract was mainly rich in the two phenylethanoids verbascoside and isoverbascoside (55\%), where the accounted amount of the former was 6 fold of that of the latter. Other important phenolic portions of the extract $(27 \%)$ were derived from the unusual flavone isoscutellarein. Thus, the compounds isoscutellarein-7-0allosyl $(1 \rightarrow 2)$ glucoside, isoscutellarein-7-0-(6-0-acetylallosyl) $(1 \rightarrow 6)$ glucoside, isoscutellarein-7-O-(6-0-acetylallosyl) $(1 \rightarrow 2)$ glucoside and its structural isomer, $4^{\prime}$-O-methylisoscutellarein-7-O-allosyl $(1 \rightarrow 2)$ glucoside and 4'-O-methylisoscutellarein-7-O-(6-0-acetylallosyl) $(1 \rightarrow 2)$ glucoside were herein described for the first time in the genus Lamium. Apigenin-7-O-glucoside, luteolin-7-O-glucoside, apigenin-7-O-rutinoside and the flavanone naringenin-7-O-rutinoside were minor constituents of this extract. Thus, overall, this work is an important contribution to

Table 4

Quantification of the identified compounds in ethanolic extract of Lamium album L.

\begin{tabular}{|c|c|c|c|}
\hline Peak & Compound & Quantified with & $\mathrm{mg} / \mathrm{g}$ extract \\
\hline 2 & Verbascoside & Verbascoside & $233.7 \pm 13.6$ \\
\hline 3 & Isoverbascoside & Verbascoside & $39.2 \pm 5.6$ \\
\hline 4 & Isoscutellarein-7-O-allosyl $(1 \rightarrow 2)$ glucoside & Apigenin-7-O-glucoside & $26.8 \pm 5.3$ \\
\hline 5 & Isoscutellarein-7-O-(6-O-acetylallosyl $)(1 \rightarrow 6)$ glucoside & Apigenin-7-O-glucoside & $23.6 \pm 6.7$ \\
\hline 6 & Luteolin-7-O-glucoside & Luteolin-7-O-glucoside & $29.7 \pm 2.2$ \\
\hline 7 & Isoscutellarein-7-O-(6-O-acetylallosyl $)(1 \rightarrow 2)$ glucoside isomer & Apigenin-7-O-glucoside & $9.6 \pm 0.3$ \\
\hline 8 & Apigenin-7-O-glucoside & Apigenin-7-O-glucoside & $16.1 \pm 5.8$ \\
\hline 9 & Isoscutellarein-7-O-(6-O-acetylallosyl $)(1 \rightarrow 2)$ glucoside & Apigenin-7-O-glucoside & $37.4 \pm 4.4$ \\
\hline 10 & 4'-O-Methylisoscutellarein-7-O-allosyl $(1 \rightarrow 2)$ glucoside & Apigenin-7-O-glucoside & $16.6 \pm 6.5$ \\
\hline 11 & 4'-O-Methylisoscutellarein-7-O-(6-O-acetylallosyl $)(1 \rightarrow 2)$ glucoside & Apigenin-7-O-glucoside & $19.4 \pm 5.2$ \\
\hline 12 & Apigenin-7-O-rutinoside & Apigenin-7-O-glucoside & $16.2 \pm 4.7$ \\
\hline 13 & Naringenin-7-O-rutinoside & Naringenin-7-O-glucoside & $32.6 \pm 5.6$ \\
\hline
\end{tabular}

Mean values \pm standard deviations. 
the chemical characterization of the L. album emphasizing that its main phenolic constituents are important antioxidant agents (verbascoside, isoverbascoside and isoscutellarein derivatives) which have been associated with diverse beneficial effects on human health. Further work is now being undertaken by our group in order to evaluate the relation of these phenolic constituents with the antioxidant capacity of L. album. We expect that if positive relations are established, consumers' and the food industry's interest in this plant will be raised.

\section{Acknowledgments}

The authors acknowledge the financial support provided by the FCT to CERNAS (project PEst-OE/AGR/UI0681/2011) and of the FCT as well as FSE (III Quadro Comunitário de Apoio) to QOPNA (project PEst-C/QUI/UI0062/2011), REDE/1504/REM/2005 (that concerns the Portuguese Mass Spectrometry Network) and the Portuguese National NMR Network (RNRMN). Olívia R Pereira was supported by a PhD grant (SFRH/PROTEC/49600/2009).

\section{References}

Albach, D. C., Grayer, R. J., Jensen, S. R., Ozgokce, F., \& Veitch, N. C. (2003). Acylated flavone glycosides from Veronica. Phytochemistry, 64(7), 1295-1301.

Baranov, A. I. (1967). Wild vegetables in Manchuria. Economic Botany, 21, 140-155.

Budzianowski, J., \& Skrzypczak, L. (1995). Phenylpropanoid esters from Lamium album flowers. Phytochemistry, 38(4), 997-1001.

Celnet (2005). Celnet recipes: Wild food guide for white deadnettle (Lamium album) Retrieved from http://www.celtnet.org.uk/recipes/

Clifford, A. W. (2001). Mediterranean vegetables: A cook's ABC of vegetables and their preparation. Boston, Massachusetts: The Harvard Common Press.

Cuyckens, H., \& Claeys, M. (2004). Mass spectrometry in the structural analysis of flavonoids. Journal of Mass Spectrometry, 39(4), 1-15.

Denisow, B., \& Bozek, M. (2008). Blooming and pollen production of two Lamium L. species. Journal of Apicultural Science, 52(1), 21-30.

Ermer, J., \& Miller, J. M. (2005). Method validation in pharmaceutical analysis: A guide to best practice. Weinheim: WILEY-VCH Verlag GmbH \& Co.

Ferreres, F., Llorach, R., \& Gil-Izquierdo, A. (2004). Characterization of the interglycosidic linkage in di-, tri-, tetra- and pentaglycosylated flavonoids and differentiation of positional isomers by liquid chromatography/electrospray ionization tandem mass spectrometry. Journal of Mass Spectrometry, 39(3), 312-321.

Fu, G., Pang, H., \& Wong, Y. H. (2008). Naturally occurring phenylethanoid glycosides: Potential leads for new therapeutics. Current Medicinal Chemistry, 15(25), 2592-2613.

Gabrieli, C. N., Kefalas, P. G., \& Kokkalou, E. L. (2005). Antioxidant activity of flavonoids from Sideritis raeseri. Journal of Ethnopharmacology, 96(3), 423-428.

Galvez, M., Martin-Cordero, C., Houghton, P. J., \& Ayuso, M. J. (2005). Antioxidant activity of Plantago bellardii All. Phytotherapy Research, 19(12), 1074-1076.

Harford, R. (2007). Eat weeds: Wild food guide to the edible plants of Britain. Retrieved from http://www.eatweeds.co.uk/

Heinrich, M., Müller, W. E., \& Galli, C. (2006). Local Mediterranean food plants and nutraceuticals, Vol. 59, Forum Nutr. Basel, Karger.

Innocenti, M., Michelozzi, M., Giaccherini, C., Ieri, F., Vincieri, F. F., \& Mulinacci, N. (2007). Flavonoids and biflavonoids in Tuscan berries of Juniperus communis L.: Detection and quantitation by HPLC/DAD/ESI/MS. Journal of Agricultural and Food Chemistry, 55(16), 6596-6602.

Isacchi, B., Iacopi, R., Bergonzi, M. C., Ghelardini, C., Galeotti, N., Norcini, M., et al. (2011). Antihyperalgesic activity of verbascoside in two models of neuropathic pain. Journal of Pharmacy and Pharmacology, 63(4), 594-601.

Karioti, A., Bolognesi, L., Vincieri, F. F., \& Bilia, A. R. (2010). Analysis of the constituents of aqueous preparations of Stachys recta by HPLC-DAD and HPLC-ESI-MS. Journal of Pharmaceutical and Biomedical Analysis, 53(1), 15-23.

Korkina, L. G. (2007). Phenylpropanoids as naturally occurring antioxidants: From plant defense to human health. Cellular and Molecular Biology, 53(1), 15-25.

Kostyuk, V. A., Potapovich, A. I., Suhan, T. O., de Luca, C., \& Korkina, L. G. (2011). Antioxidant and signal modulation properties of plant polyphenols in controlling vascular inflammation. European Journal of Pharmacology, 658(2-3), 248-256.
Kupeli, E., Sahin, F. P., Yesilada, E., Calis, I., \& Ezer, N. (2007). In vivo anti-inflammatory and antinociceptive activity evaluation of phenolic compounds from Sideritis stricta. Zeitschrift fur Naturforschung. C, Journal of Biosciences, 62(7-8), 519-525.

Li, L., Liu, C. M., Liu, Z. Q., Tsao, R., \& Liu, S. Y. (2009). Identification of phenylethanoid glycosides in plant extract of Plantago asiatica L. by liquid chromatographyelectrospray ionization mass spectrometry. Chinese Journal of Chemistry, 27(3), 541-545.

Luczaj, L. (2008). Archival data on wild food plants used in Poland in 1948. Journal of Ethnobiology and Ethnomedicine, 4, 4.

Matkowski, A., \& Piotrowska, M. (2006). Antioxidant and free radical scavenging activities of some medicinal plants from the Lamiaceae. Fitoterapia, 77(5), 346-353.

Mihaly Cozmuta, A., Bretan, L., Mihaly Cozmuta, L., Nicula, C., \& Peter, A. (in press). Lead traceability along soil-melliferous flora-bee family-apiary products chain. Journal of Environmental Monitoring, doi:10.1039/C2EM30084B.

Morikawa, T., Pan, Y., Ninomiya, K., Imura, K., Matsuda, H., Yoshikawa, M., et al. (2010). Acylated phenylethanoid oligoglycosides with hepatoprotective activity from the desert plant Cistanche tubulosa. Bioorganic \& Medicinal Chemistry, 18(5), 1882-1890.

Nagai, T., Miyaichi, Y., Tomimori, T., Suzuki, Y., \& Yamada, H. (1992). In vivo antiinfluenza virus activity of plant flavonoids possessing inhibitory activity for influenza virus sialidase. Antiviral Research, 19(3), 207-217.

Ninomiya, K., Nishida, S., Matsura, Y., Asada, M., Kawahara, Y., Yoshikawa, M., et al. (2006). Fat-metabolism improving agent for use in food/drink for improving fat metabolism and preventing/treating lifestyle related disease e.g. diabetes, contains polar solvent extract of herb e.g. rose hip fruit, mugwort or safflower. In MORISHITA JINTAN KK, (MORI-Non-standard) (pp. 19).

Paduch, R., Matysik, G., Wojciak-Kosior, M., Kandefer-Szerszen, M., Skalska-Kaminska, A., Nowak-Kryska, M., et al. (2008). Lamium album extracts express free radical scavenging and cytotoxic activities. Polish Journal of Environmental Studies, 17(4), 569-580.

Paduch, R., Wójciak-Kosior, M., \& Matysik, G. (2007). Investigation of biological activity of Lamii albi flos extracts. Journal of Ethnopharmacology, 110(1), 69-75.

Pereira, O. R., Silva, A. M. S., Domingues, M. R. M., \& Cardoso, S. M. (2012). Identification of phenolic constituents of Cytisus multiflorus. Food Chemistry, 131(2), 652-659.

Petreska, J., Stefova, M., Ferreres, F., Moreno, D. A., Tomás-Barberán, F. A., Stefkov, G., et al. (2011). Potential bioactive phenolics of Macedonian Sideritis species used for medicinal "Mountain Tea". Food Chemistry, 125(1), 13-20.

Sahin, F. P., Ezer, N., \& Calis, I. (2006). Terpenic and phenolic compounds from Sideritis stricta. Turkish Journal of Chemistry, 30(4), 495-504.

Saracoglu, I., Harput, U. S., \& Ogihara, Y. (2004). Acylated flavone glycosides from Veronica pectinata var. glandulosa and V-persica. Turkish Journal of Chemistry, 28(6), 751-759.

Singleton, V. L., \& Rossi, J. A. (1965). Colorimetry of total phenolics with phosphomolybdicphosphotungstic acid reagents. American Journal of Enology and Viticulture, 16, 144-158.

Snyder, L., Kirkland, J., \& Dolan, J. (2010). Introduction to modern liquid chromatography. New Jersey: John Wiley \& Sons, Inc..

Sturtevants, E. L. (1919). Edible plants of the world. (Vol.). : The Southwest School of Botanical Medicine.

Tomás-Barberán, F. A., Francisco, A., Gil, M. I., Ferreres, F., \& Tomás-Lorente, F. (1992). Flavonoid p-coumaroylglucosides and 8-hydroxyflavone allosylglucosides in some labiatae. Phytochemistry, 31(9), 3097-3102.

Tomás-Barberán, F. A., Grayer-Barkmeijer, R. J., Gil, M. I., \& Harborne, J. B. (1988). Distribution of 6-hydroxy-, 6-methoxy- and 8-hydroxyflavone glycosides in the labiatae, the scrophulariaceae and related families. Phytochemistry, 27(8), 2631-2645.

Turner, N. J., Luczaj, L. J., Migliorini, P., Pieroni, A., Dreon, A. L., Sacchetti, L. E., et al. (2011). Edible and tended wild plants, traditional ecological knowledge and agroecology. Critical Reviews in Plant Sciences, 30(1-2), 198-225.

Valyova, M. S., Dimitrova, M. A., Ganeva, Y. A., Mihova Kapchina-Toteva, V., \& Petkova Yordanova, Z. (2011). Evaluation of antioxidant and free radical scavenging potential of Lamium album L. growing in Bulgaria. Journal of Pharmacy Research, 4(4), 945-947.

$\mathrm{Xu}, \mathrm{F}$. (2008). Chinese medicine e.g. for treating arthropathy, comprises broad cocklebur, vervain, condyle grass, motherwort, saxifrage, cactus, mulberry branch, white dead nettle, Boston ivy, Folium photiniae, water pepper and Chinese fever vine. In XU F, (XUFF-Individual) (pp. 10).

Yalcin, F. N., \& Kaya, D. (2006). Ethnobotany, pharmacology and phytochemistry of the Genus Lamium (Lamiaceae). FABAD Journal of Pharmaceutical Sciences, 31, 43-52.

Yang, H., Protiva, P., Cui, B. L., Ma, C. Y., Baggett, S., Hequet, V., et al. (2003). New bioactive polyphenols from Theobroma grandiflorum ("Cupuacu”). Journal of Natural Products, 66(11), 1501-1504

Yoon, K. D., Jeong, D. G., Hwang, Y. H., Ryu, J. M., \& Kim, J. (2007). Inhibitors of osteoclast differentiation from Cephalotaxus koreana. Journal of Natural Products, 70(12), 2029-2032. 